\title{
Sickle Cell Disease in Pregnancy: Active Nursing Management
}

\author{
Article by Anisley Fabars-Johnson \\ BSN, Texila American University, Saint Kitts and Nevis \\ E-mail: anisley30@yahoo.com
}

\begin{abstract}
Sickle cell disease is considered as a major complication and risk factor for perinatal morbidity Imortality. Literature document that most pregnancies complicated by sickle cell are likely to result in live birth, but the consequences of influence of the disease for the pregnancy/newborn remains a significant concern for health care providers worldwide. According to the bibliography obstetrical-fetal risks are due to the metabolic demands, hypercoagulable state, and vascular stasis associated with pregnancy characterized normally for blood cells to be able to carry oxygen to the growing fetus. With sickle cell anemia, the abnormal red blood cells and anaemic characteristics of the disease physiopathology may result in lower amounts of oxygen going to the developing baby with negative outcome for the future newborn.

Research review studies agreed that access of the pregnant client to a multidisciplinary care team knowledgeable about sickle cell disease and high-risk obstetrics can significantly decrease fetomaternal morbidity and mortality. Example: decreases in spontaneous miscarriage, in perinatal death rates and lowered incidence of preterm labour. Active prenatal management include: education; genetic counselling and prenatal diagnosis for couples at risk; improving nutritional status; vaccination for disease prevention, and early detection of bacterial infection.

Objective of this study was to explore active nursing management of the pregnant women with sickle cell disease, including education, treatment and nursing intervention.

Method: use of English Literature review current through: Jun 2017, Data were searched using MEDLINE, EMBASE, PUBMED and COCHRANE Systematic Reviews.
\end{abstract}

Keywords: Sickle Cell Disease, complication, feto-maternal risk, active nursing management.

\section{Introduction}

Sickle cell disease (also called sickle cell anemia) is an inherited blood disorder that affects red blood cells. People at risk for inheriting the gene for sickle cell, are descended from people who are or were originally from Africa or parts of India and the Mediterranean. Population mobility has spread the sickle cell gene through Europe, Asia, Americas and the Caribbean. It means that millions of people have Sickle Cell Disease worldwide. They are either carriers of or have the sickle cell trait. Carriers are usually asymptomatic and have a low percentage of sickle hemoglobin ( $\mathrm{HbS})$. Two parents who are carriers can both pass on the sickle cell trait to their offspring, resulting in SCD. There is a 50\% chance with each pregnancy for the child of two sickle cell carriers to be born with the sickle cell trait, and there is a $25 \%$ chance for the child to be born with SCD.

The disease has been declared by WHO (2006) as a major world health problem. The sickle cell gene mutation causes the body to produce abnormal haemoglobin. In sickle cell disease, the haemoglobin clumps together, causing red blood cells to become stiff and develop a C-shaped ("sickle") form. These red blood cells can block blood vessels, reducing blood flow, which limit adequate oxygenation to many parts of the body. This contributes to the severe pain experienced as a sickle cell crisis and both shortterm and long-term organ damage. The disease also makes patients more susceptible to infections as the spleen may be damage decreasing the individual ability to fight infection. 
DOI: $10.21522 / \mathrm{TIJNR} .2015 .03 .02 . A r t 009$

ISSN: $2520-3126$

For some pregnant women, a less percentage of studies show no change in their disease during pregnancy, while others may have a worsening of the disease, resulting from or in many cases as the pregnancy accelerates sickle cell complications surface.

\section{Risks to the woman}

Sickle cell disease is associated with:

- Increased incidence of perinatal mortality

- Premature labour

- More acute painful crises during pregnancy

- Increase in spontaneous miscarriage

- Recurrent antenatal hospitalisation

- Maternal mortality

- Delivery by caesarean section

- Infections (especially urinary tract infection) during pregnancy

- Thromboembolic events

- Ante partum haemorrhage

- Increased risk of pre-eclampsia

- Pregnancy-induced hypertension

- Postpartum infection

- Increased numbers of cases with acute chest syndrome

- Increases the risk of blood transfusion reactions

- Increased incidences of thromboembolism and pulmonary infarct, which are usually fatal

- Heart enlargement and heart failure from anemia

- Vision problems.

\section{Risks to the baby}

Sickle cell disease is associated with fetal complications such as:

- Premature birth

- Fetal growth restriction or IUGR (intrauterine growth retardation)

- Increase the likelihood of fetal distress

- Chance of their baby being affected by Sickle cell disease

- Birth defects

- Low weight babies

- Stillbirths.

- Newborn death

- Newborn with Severe anemia.

- Neonatal jaundice.

\section{Nursing interventions}

Goals of active nursing intervention related to management of sickle cell disease in pregnancy.

- Improve obstetric and neonatal outcomes.

- Give emotional support and measures to alleviate symptoms associated with disease manifestations.

- Enhance patient knowledge for better understanding and cooperation.

- Minimize complications

- Comprise a multidisciplinary approach.

- Promote and maintain a safe environment during pregnancy, labor, delivery, and the postpartum period.

- Enhanced patient sense of self-esteem and power. 
- Active Nursing interventions for pregnant patient with sickle cell anemia should start at the client first antenatal visit.

- Early and regular prenatal care allows healthcare provider to keep a close monitoring on the disease condition and on the health of developing baby.

- Antenatal care should be provided by a multidisciplinary team including an obstetrician and midwife with experience of high-risk antenatal care and a haematologist with an interest in Sickle cell disease condition.

- A head to toe assessment should be performed at each visit to discard signs of worsening anemia, joints swelling, appropriate fundal high according to gestational age.

- Assess for fetal movement and normal fetal heart rate.

- Assess for any sign and symptoms of infection.

- Antenatal education is a significant resource in increasing knowledge for the pregnant client, her partner and family involve, about the disease process, with beneficial results for a healthy pregnancy and baby.

The nurse must teach the patient about situations that can precipitate a sickle cell crisis and steps to help prevent or diminish such crises example:

- Keep warm.

- Maintain adequate hydration.

- Avoid stressful situations.

- Educate patient on the importance of maintain a healthy diet.

- Encourage patient to take folic acid, vitamins and iron supplements.

- Enhance good patient-nurse communication and encourage patient to report any changes.

- Educate patient to maintain proper hygiene to avoid infections.

Studies have also demonstrated an increase in the incidence of urinary tract infection and asymptomatic bacteraemia so is recommended a urinalysis to be performed at each antenatal visit and midstream urine should be sent for culture and sensitivity monthly. Blood pressure and weight should be checked at each visit to monitor for signs of preeclampsia.

\section{Ultrasound scanning during pregnancy}

Serial growth scans allow early detection of fetal growth restriction and hence aid appropriate timing of delivery to reduce perinatal mortality and morbidity

- Women should be offered a viability scan at 7-9 weeks of gestation.

- Women should be offered the routine first-trimester scan (11-14 weeks of gestation) and a detailed

- Anomaly scans at 20 weeks of gestation.

- serial fetal biometry

- Growth and amniotic fluid monitoring scans every 4 weeks from 24 weeks of gestation.

- 32 weeks and advance, growth and well-being scans.

\section{This includes}

- Nonstress tests: measure fetal heart rate

- Biophysical profile tests: monitor fetal movements, muscle tone, and breathing movements, etc.

- Doppler sonography: monitor blood flow from the placenta to the fetus.

\section{Nursing intervention should focus on}

- Managing Pain

- Preventing and Managing Infection

- Promoting Coping Skills

- Monitoring and Managing Potential Complications

- Promoting Home and Community Based Care

- Medications 
DOI: $10.21522 /$ TIJNR.2015.03.02.Art009

ISSN: $2520-3126$

\section{This includes}

- Daily folic acid and prophylactic antibiotics (if not contraindicated).

- Drugs that are unsafe in pregnancy should be stopped.

- Iron supplementation should be given only if there is laboratory evidence of iron deficiency.

- Low-dose aspirin $75 \mathrm{mg}$ once daily from 12 weeks of gestation in an effort to reduce the risk of developing pre-eclampsia.

- Prophylactic low-molecular-weight heparin during antenatal hospital admissions. For the associate risk with risk of venous thromboembolism.

\section{Managing pain}

Sickle cell disease (SCD) is associated with chronic haemolysis and painful episodes, during pregnancy, SCD may become more severe, and pain episodes may happen more often; usually happen in the bones, joins, abdominal organs. They can last a few hours, few days, or last for weeks. Sickle cell painful crisis is the number one cause of recurrent hospitalization. It must be treated with medications that are safe to use during pregnancy.

Women admitted with sickle cell crisis should be looked after by the multidisciplinary team, involving obstetricians, midwives, haematologists and anaesthetists. A detail assessment should be rapidly carried out to rule out medical complications requiring intervention such as ACS, sepsis or dehydration.

- Dehydration and electrolyte imbalance caused by vomiting, diarrhoea or pyrexia should by corrected with the administration of intravenous fluid.

- Initial investigations should include full blood count, reticulocyte count and renal function. Other investigations will depend on the clinical scenario but may include blood cultures, chest X-ray, urine culture and liver function tests.

- Oxygen therapy may be required if baseline less than $95 \%$ for supplementary therapy and to prevent fatal hypoxia. Sickle cell disease can lead to severe placental damage which decreases the transfer of blood rich in oxygen and nutrient to the fetus.

The World Health Organization analgesic ladder should be used, starting with paracetamol for mild pain; NSAIDs can be used for mild to moderate pain between 12 and 28 weeks of gestation. Weak opioids such as co-dydramol, co-codamol or dihydrocodeine can be used for moderate pain, and stronger opiates such as morphine can be used for severe pain. Morphine or diamorphine can be given by the oral, subcutaneous, intramuscular or intravenous route depending on the woman's preference and local expertise. Parenteral opiates can be given by intermittent bolus or patient-controlled administration systems.

Pethidine should be avoided because of the risk of toxicity and pethidine-associated seizures in patients with SCD. While women are receiving parenteral opiates, they should be nursed in an area where they can undergo hourly observations .Assessments of pain score, sedation score and oxygen saturation should be performed. Opiate morphine constricts the blood vessels in the placenta and so may harmful to the fetus. Nurses must monitor continuo fetal activity to rule out fetal distress. And provision of social, physical and psychological support to alleviate symptoms associated with chronic pain.

According to the literature review sickle cell pain crisis on third trimester are more likely lasts for a longer time or until after delivery. Which required a longer stay in hospital, in most cases until the baby is born.

- The nurse must monitor patient for sings of worsening condition:

- A fever higher than $101^{\circ} \mathrm{F}$

- Difficulty breathing

- Chest pain

- Abdominal oedema

- A severe headache 
- A sudden feeling of weakness

- Seizures

- Sudden vision loss

\section{Blood transfusion during pregnancy}

A systematic cohort review indicated that there is insufficient evidence to draw conclusions about the role of prophylactic transfusion in pregnancy to decrease the incidence of maternal painful crises. Routine prophylactic transfusion is not recommended during pregnancy for women with SCD. If acute exchange transfusion is required for the treatment of a sickle complication, it may be appropriate to continue the transfusion regimen for the remainder of the pregnancy. Risks associated with transfusion, include alloimmunisation (the formation of antibodies to red cell antigens), delayed transfusion reactions, transmission of infection and iron overload.

\section{Blood transfusion therapy should be given only}

1. To compensate for anemia with symptoms of impending cardiac failure

2. To provide a prophylactic "top-up" transfusion before a caesarean section

3. Emergency transfusion for acute anemia $(<5 \mathrm{~g} / \mathrm{dL}$ hemoglobin)

4. Twin pregnancy

5. Previous history of perinatal mortality

6. Septicaemia,

7. Acute renal failure

8. Acute chest syndrome

9. A recent neurologic event, hypoxemia,

Randomized studies have shown that many patients, when closely monitored, had well-tolerated anemia, regardless of the level of hemoglobin, and that many were able to complete their pregnancy successfully without transfusion, regardless of the route of delivery.

\section{Recommendations}

\section{Preconception care}

- Amplify the role of family planning and health promotion in the communities to provide education to all women in childbearing age about Sickle cell disease effect- outcome in pregnancy. And the importance of have the haemoglobinopathy status of their partner before becoming pregnant.

- Promote counselling about reproductive options, planning and contraceptive choice.

- Advice the couple to assist genetic counselling.

- Folic acid (5 mg) should be given once daily both preconceptual and throughout pregnancy.

\section{Antenatal care}

- Identify the client as a high risk and refer couple to high risk pregnancy clinic.

- Remit patient to a high - risk clinic to be evaluate by a multidisciplinary team including an obstetrician and midwife with experience of high-risk antenatal care and a haematologist with an interest in SC.

- Promote screening programme to ensure that screening tests are offered by $8-10$ weeks of pregnancy by Primary care or maternity services.

- To monitor and prevent worsening of the condition.

- Iron supplementation should be given only if there is laboratory evidence of iron deficiency.

- Referring to type of delivery is important to maintain proper temperature on delivery room to avoid hypothermia, acidosis and hypoxemia. And to prevent vaso-occlusion and joint pain

- Care could be improved by more specialist centres and specialist nurses or midwives. 
DOI: $10.21522 /$ TIJNR.2015.03.02.Art009

ISSN: $2520-3126$

- Guidelines need to be created for all healthcare professionals to improve management of these women during pregnancy.

- Refer to genetic counselling and promote partner screening testing to determine presence of HbS.

\section{Conclusion}

The detail of appropriate prenatal care and perinatal management for these patients is still a matter for debate in developed countries. However nowadays outcomes have improved significant for pregnant women with Sickle Cell Disease and newborns thanks to medical/obstetric advancement and earlyvigilant nursing care, with a good chance of having a safe and healthy pregnancy. Different studies agreed for the need of a dedicated multidisciplinary health care for pregnant women and newborn with SCD for crucial improvement of the disease condition and prevention of short/long term complications. Optimal management during pregnancy should be directed to education/counselling about healthy diet, prenatal vitamins, folic acid supplements and B vitamins, prevention of dehydration, also avoidance of precipitants such as a cold environment and excessive exercise, that can trigger pain crises, and to prevent chronic organ damage, and optimization of fetal health with the goal of minimizing early maternal mortality.

\section{References}

[1]. Eugene Oteng-Ntim, Daveena Meeks, Paul T Seed, Louise Webster, Jo Howard, Pat Doyle, and Lucy C Chappell. Adverse maternal and perinatal outcomes in pregnant women with sickle cell disease: systematic review and meta-analysis. Blood, March 2015 DOI: 10.1182/blood-2014-11-607317

[2]. Goldsmith JC, Bonham VL, Joiner CH, et al. framing the research agenda for sickle cell trait: building on the current understanding of clinical events and their potential implications. Am J Hematol 2012; 87:340.

[3]. NHLBI. Evidence-Based Management of Sickle Cell Disease: Expert Panel Report, 2014. p.24. http://www.nhlbi.nih.gov/guidelines (Accessed on August 11, 2014).

[4]. Oteng-Ntim E, Meeks D, Seed PT, Webster L, Howard J, Doyle P, Chappell LC. Adverse maternal and perinatal outcomes in pregnant women with sickle cell disease: systematic review and meta-analysis. Blood. 2015; 125(21):3316-25. [PubMed]

[5]. Oteng-Ntim E, Ayesha B, Knight M, Howard J. Pregnancy outcome in patients with sickle cell disease in the UK--a national cohort study comparing sickle cell anaemia (HbSS) with HbSC disease. Br J Haematol 2015; 169:129.

[6]. Pintova S, Cohen HW, Billet HH. Sickle cell trait: is there an increased VTE risk in pregnancy and the postpartum? PLoS One 2013; 8:e64141.

[7]. Royal College of Obstetricians and Gynaecologists Green-top. Guideline Management of sickle cell disease in pregnancy. RCOG. 2011; 61:1-20.

[8]. Souza J.P., Cecatti J.G., Fagundes A., Morais S.S., Villar J., Carroli G.A. Maternal near miss and maternal death in the World Health Organization's 2005 global survey on maternal and perinatal health. Bull World Health Org. 2010; 88:113-119. [PubMed].

[9]. Yawn BP, Buchanan GR, Afenyi-Annan AN, et al. Management of sickle cell disease: summary of the 2014 evidence-based report by expert panel members. JAMA 2014; 312:1033. 


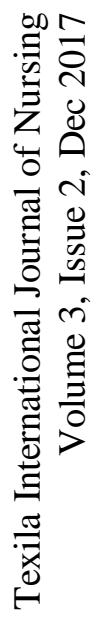

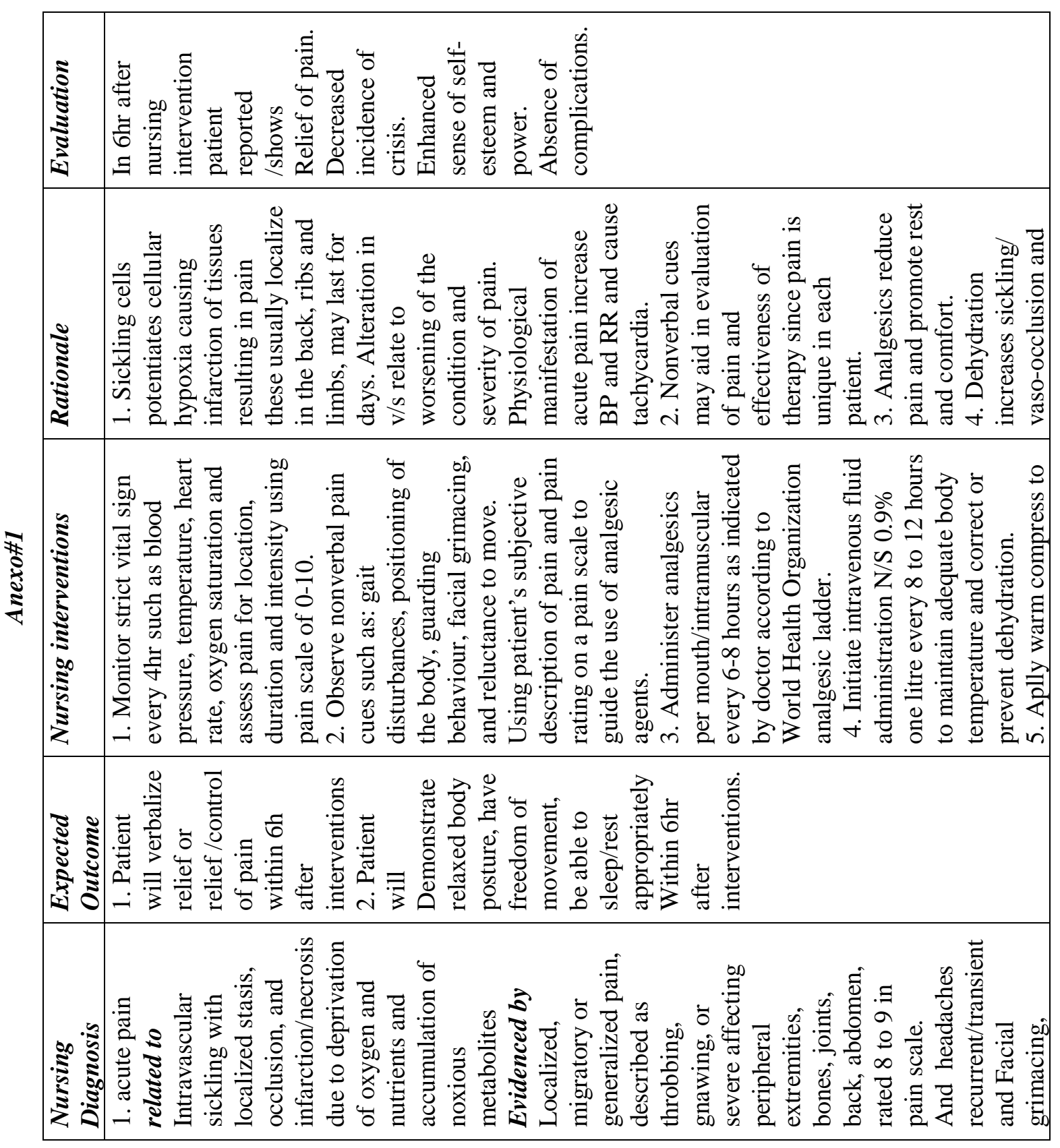




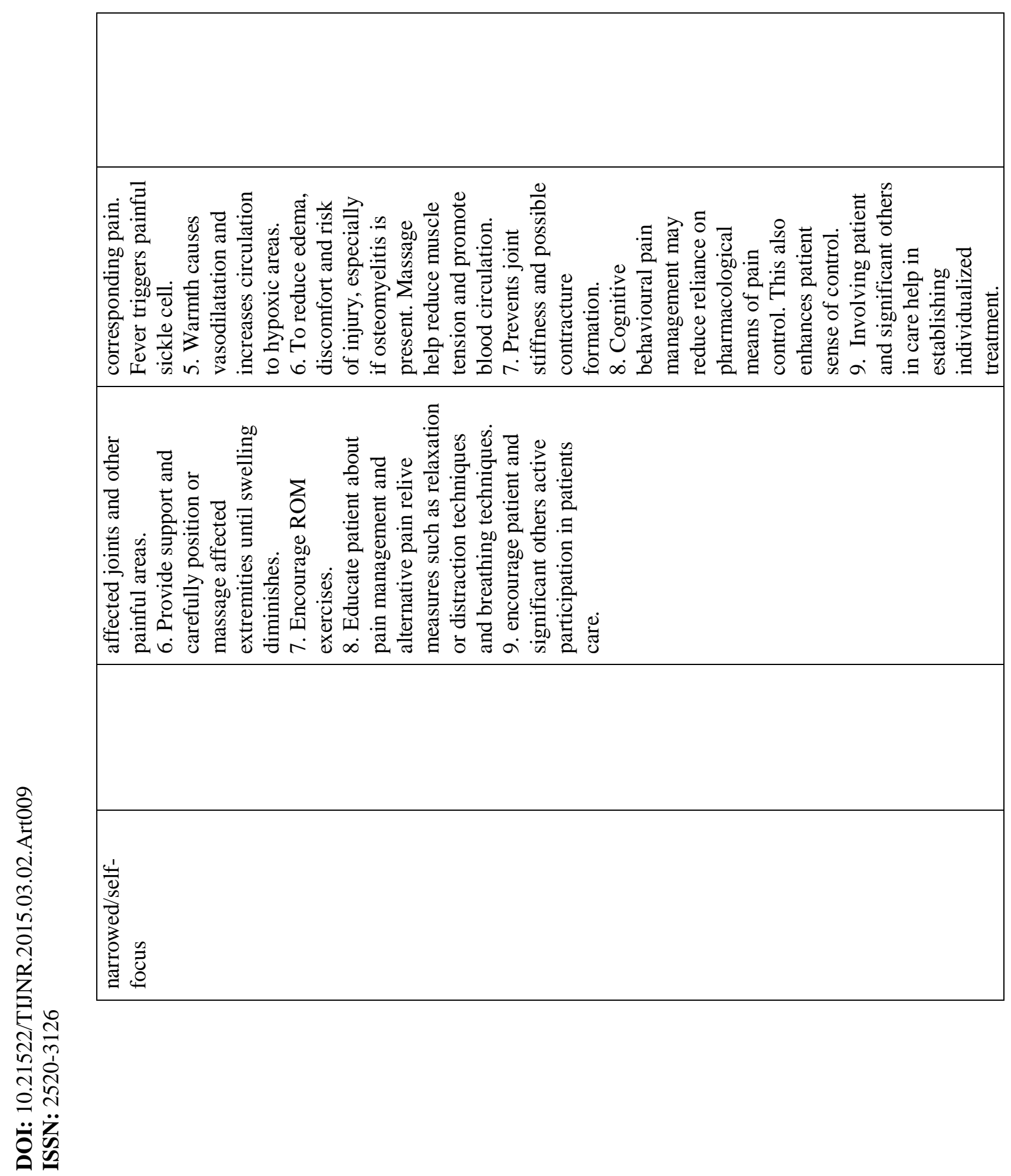




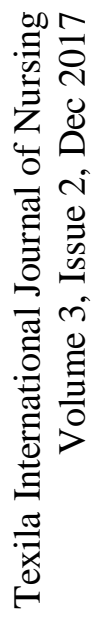

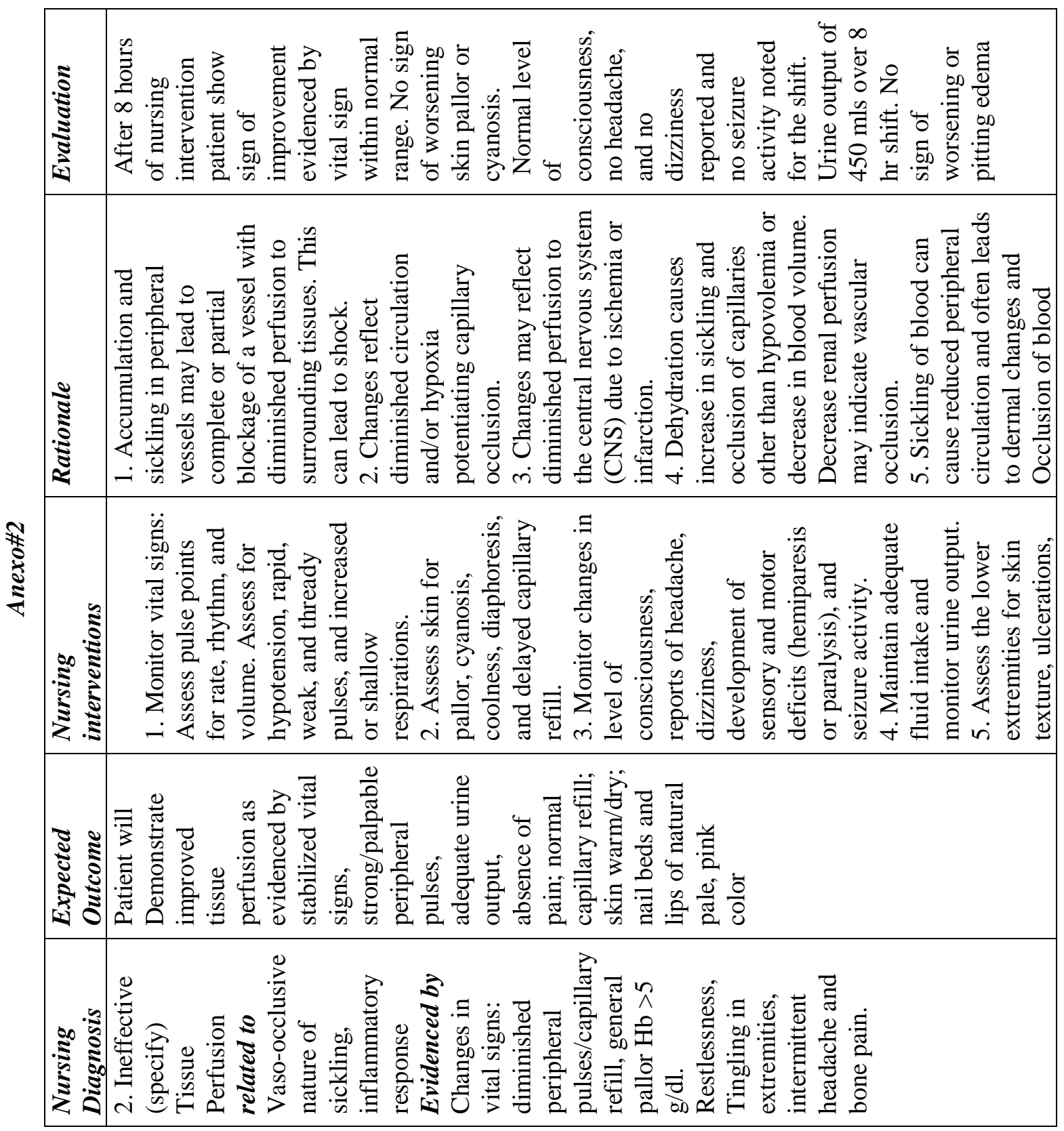




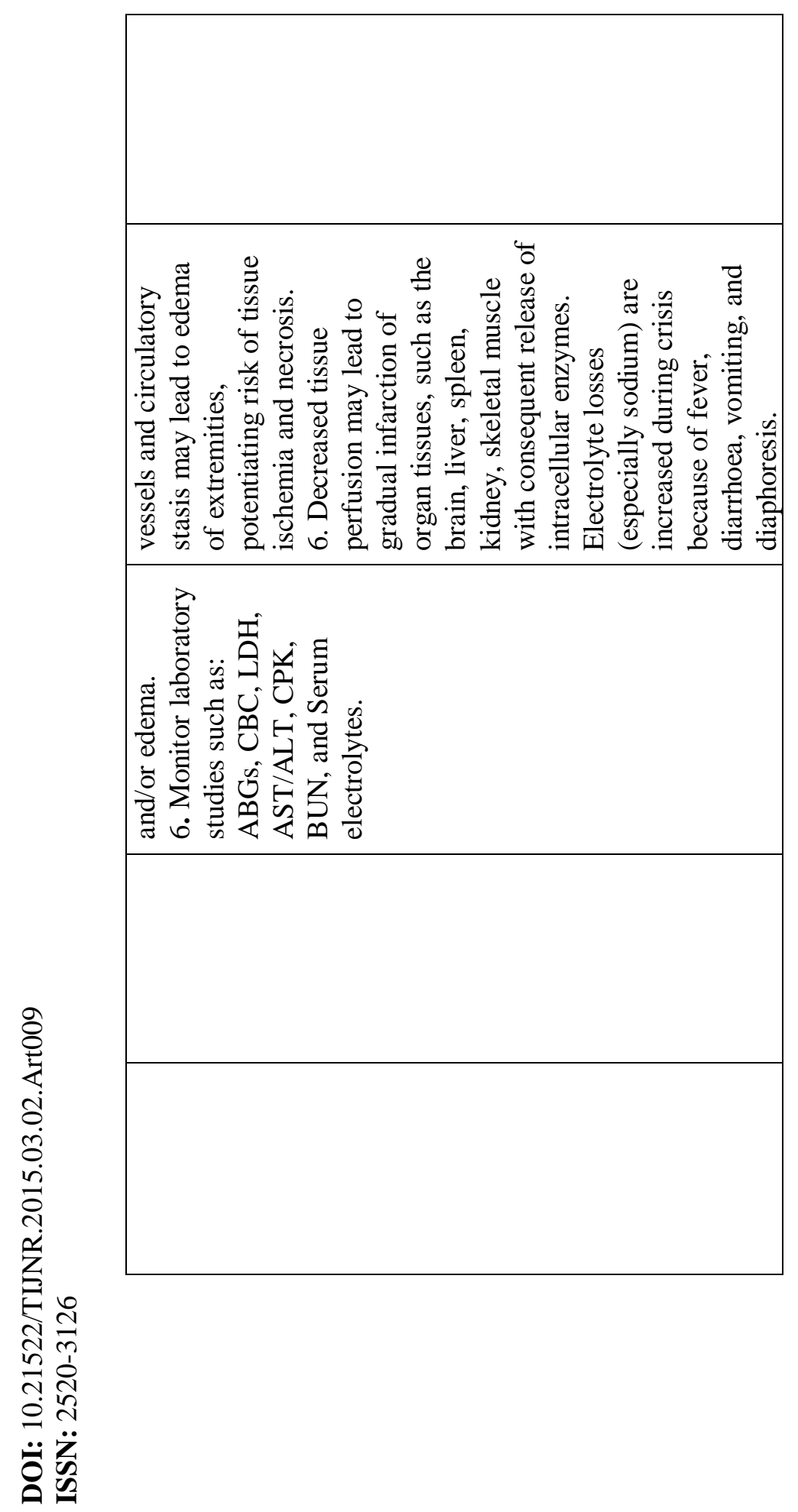




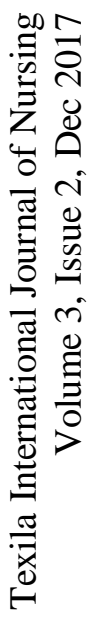

\begin{tabular}{|c|c|}
\hline 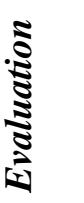 & 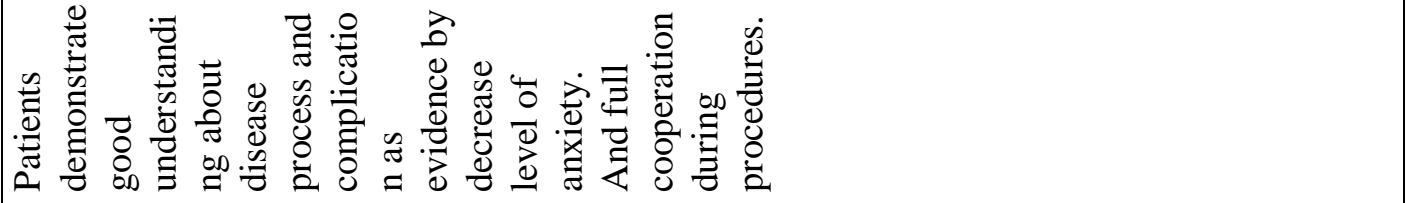 \\
\hline 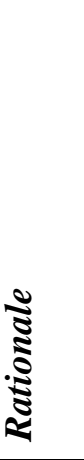 & 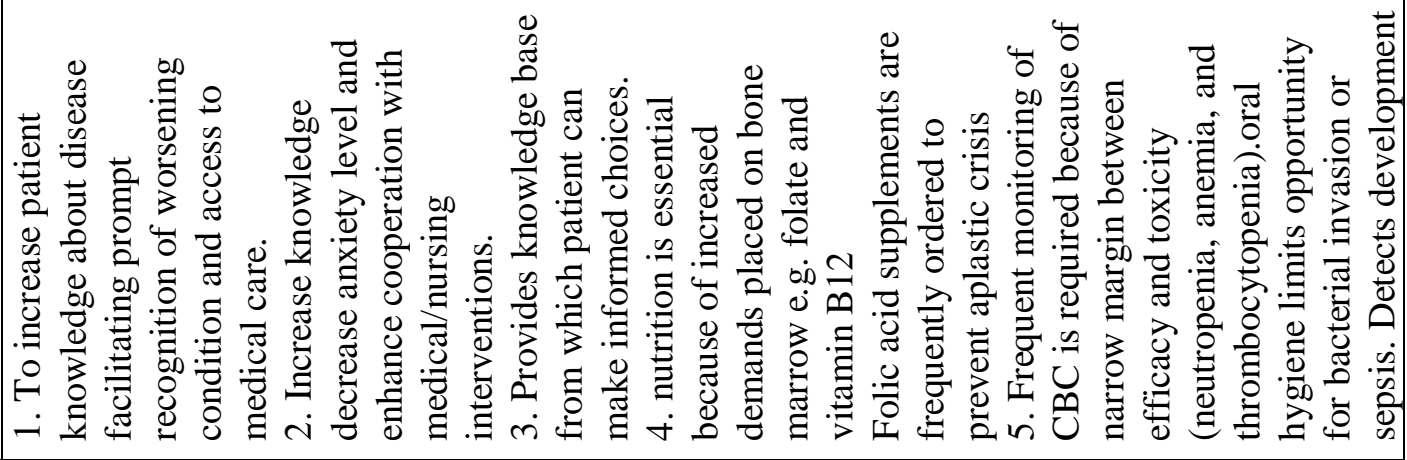 \\
\hline 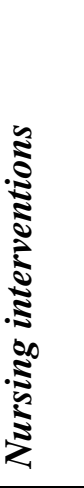 & 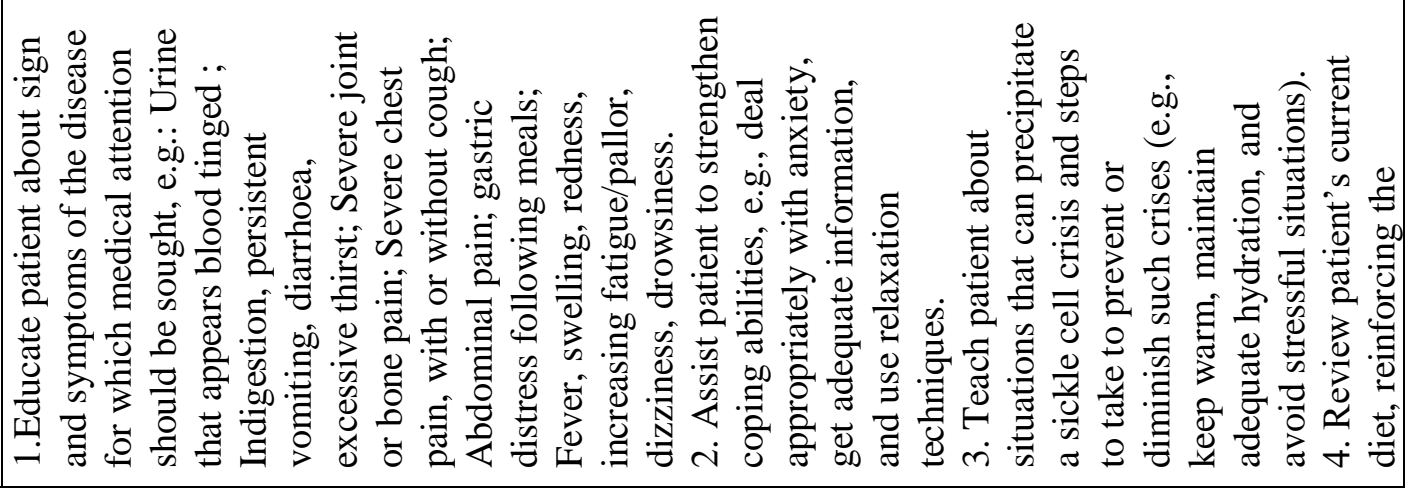 \\
\hline 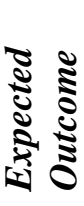 & 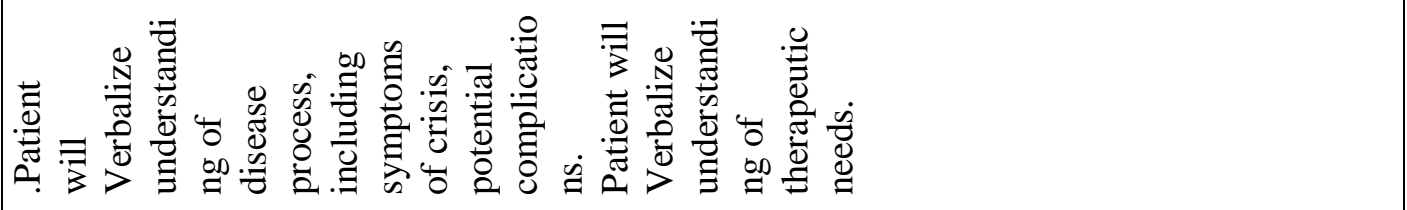 \\
\hline 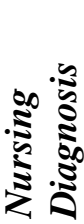 & 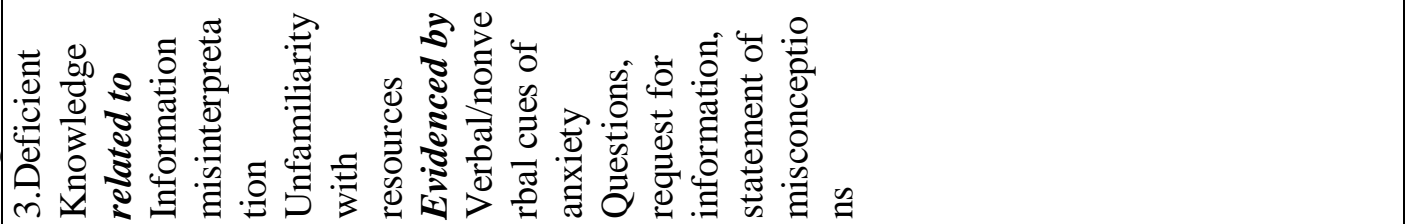 \\
\hline
\end{tabular}




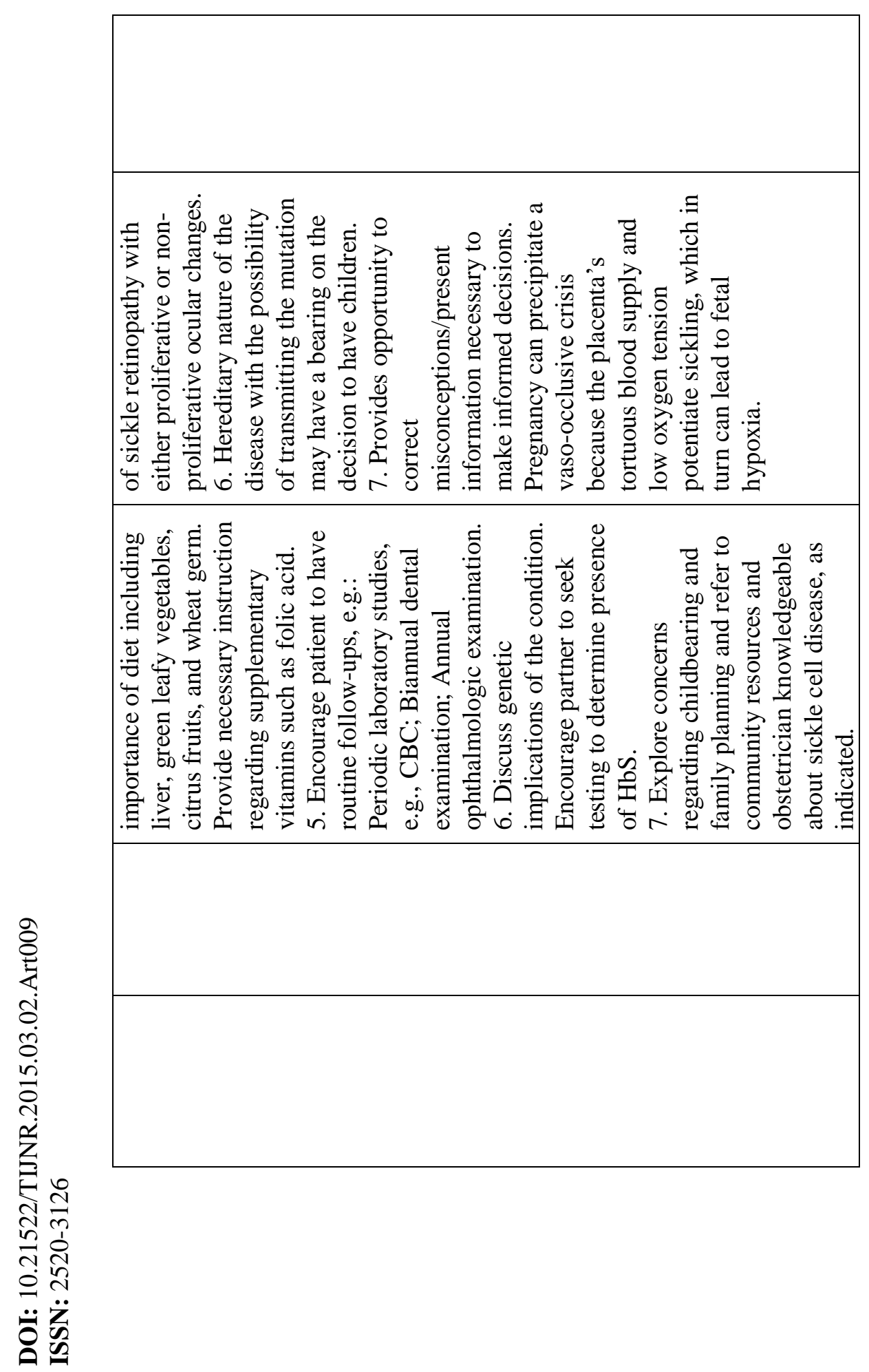

